\title{
Indian Sign Language Recognition Based on Gray Level Co-occurrence Matrix and 7Hu Moment
}

\author{
Umang Patel \\ PG Student, dept of EXTC \\ DJSCE, Vile Parle \\ Mumbai, India
}

\author{
Aarti G. Ambekar \\ Assistant Prof, dept of EXTC \\ DJSCE, Vile Parle \\ Mumbai, India
}

\begin{abstract}
Communication is an important part of our day to day life. But it is very challenging for normal people to communicate with deaf, dumb \&blind people \& vice versa. This is because of a deaf, dumb need sign language for communication and normal people cannot understand sign language easily. Therefore there is a demand of converting and translating sign languages. This paper removes the barrier of communication between them. In this paper, hand gestures are captured, processed and then translated into speech \& text. In the proposed method, two languages for a character as well as words are chosen namely English \& Hindi. Feature extraction is done using moment technique and gray level co-occurrence matrix. Two classification techniques (PNN \& KNN) are used $\&$ performance parameters are compared between both classifiers.
\end{abstract}

\section{General Terms}

Hand Gesture into Speech \& Text, Deaf, Dumb \& Blind People, Sign Language

\section{Keywords}

Hand Gesture, Sign Recognition, Image Processing, Indian Sign Language (ISL), 7Hu Moments, KNN Classifier, PNN Classifier, GLCM.

\section{INTRODUCTION}

In our day to day life, communication plays crucial role for convey information from one person to another person. But it becomes very tough for the people who are deaf and dumb to communicate with normal people. Sign language is the only one way which connects them. But normal people are unfamiliar of sign language. So there is only one way and that is to covert and translates sign language into text \& speech. It is known as sign recognition. Sign language is a mixer of body languages, hand gestures and facial expressions. Among those, hand gestures is the most effective method hence majority of the research is going on decoding the hand gestures [1]. There are two approaches for decoding the hand gestures as listed below.

\section{a) Image Based \\ b) Sensor Based}

From the listed methods, sensor based method is a very complicated method as it needs hardware and sensor based hand gloves and also troubleshooting is very difficult so there is a need of high skilled person. While image based method is relatively very easy to implement, decode and use. Therefore most of the research is done on this method. There are many sign languages used worldwide, which differs from each other. For example: In India ISL (Indian sign language), In America, ASL (American Sign Language), likewise there are many sign. Language such as FSL, RSL \& so on. Proposed work considers Indian sign languages (ISL) and American Sign Language (ASL). There are over 615 languages used in India based on culture \& regions, as a result languages used for sign recognition are also different. Sign language is also classified as one handed \& double handed. Over here double handed sign language is used for sign recognition. The method which is proposed in this paper uses a web camera for capturing gestures and then it will pre-process by MATLAB. After having the processed image in MATLAB, next step is feature extraction \&then processed by classifier. Finally recognized gestures are displayed as Hindi \& English text and also sound files of respective gestures are available.

Proposed method, consider a single character and words in both English and Hindi sign languages. In single character, alphabets of each individual language are used. While words which are used more frequently in day to day life and most common for communication such as 'Hello', 'Stop', 'Hi', Days of week etc. has only single gestures for recognition. This is known as single word using single gesture (SWSG). Words which are uncommon \& rarely used for communication such as name of the person, random words are unable to represent in single gestures. For those kinds of words, one need to recognize each letter of word by respective alphabet so multiple gestures need to be decoded to recognize the entire word. This is known as single word using multiple gestures (SWMG).

\section{LITERATURE SURVEY}

Mostly sign language recognition is done by two approaches image based and sensor based approach [8]. Currently Imagebased method is studied in research area [1]. Sign language translator smoothly implemented on the smartphone platform with the help of image based method, due to its flexibility, mobility and ease of use [1]. In this sign language, gestures captured by camera are translated into text. Developing a system without hand gloves and sensors and only by capturing the gesture movements rapidly and converting them into voice and vice versa, it makes the communication easy for hearing and speech impaired people. Double handed Indian sign language is captured in a series of gestures and it is processed with the help of MATLAB and then it is translated into voice and text. Indian sign language recognition using method of orthogonal transform has a disadvantage of more computation speed.

While capturing, extracting \& preprocessing images following parameter helps to improve a result. That parameter are lighting changes, different sizes \& shapes of users hand, 
background, skin color, distance from camera, angle position of hand.

\section{HINDI SIGN LANGUAGE}

In India approximate $6.75 \%$ of population is of hearing and speech impaired people. In the country like India, where rate of ability to read \& write is very less [8], therefore it is very tough for hearing impaired people to interact with normal people. In India, there are many regional languages as per region and states. So there is not a common Indian sign language, it differs from place to place in India. But as Hindi is the national language of India, it's known in most part of India. Keeping all this things into mind in the proposed method two languages are considered for sign recognition namely, English and Hindi.

\section{PROPOSED METHOD}

The block diagram shown in Fig 1 is proposed method for extracting gestures and translates them into text \& speech of both languages Hindi \& English for character \& word recognitions.

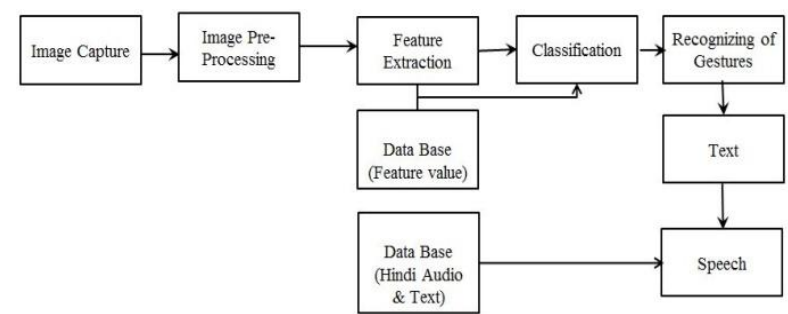

Fig 1: Block diagram of Proposed Method

\subsection{Image Capture}

In sign recognition, first step is capturing a gesture. Interfacing a camera is very crucial work. To capture the hand gesture, web camera is used. Now a day's web camera is inbuilt in laptops or one can use external camera for interfacing. Captured images should be in good quality. So selection of good webcam \& its interfacing is an important task of this method.

\subsection{Image Preprocessing}

Image preprocessing contains filtering, cropping, brightness adjustment, contrast adjustment, skin segmentation, binary conversion \& many more. To do such process concept of image cropping, image enhancement\& image segmentation are required.

Captured images are in the form of RGB. So the first step is to convert RGB images to binary images. To remove unwanted information from captured image, cropping of image is to be done. To improve quality of image, enhancement can be done in certain selected area. In image segmentation, edge detection \& skin segmentation method is used. Edge detection used for detecting the boundary of images. Skin segmentation helps to remove complex background. Those all pre-processing techniques which helps for evaluating features values.

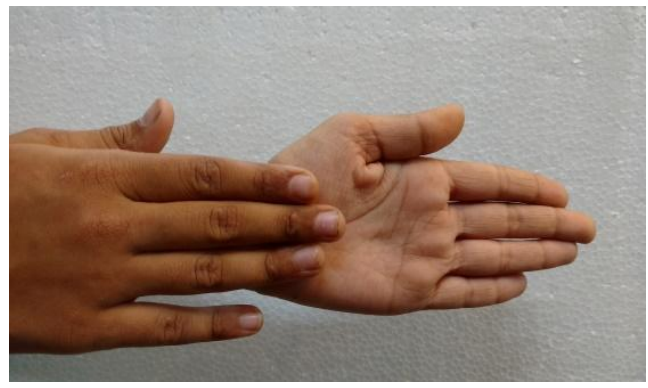

Fig 2: Captured Image

\subsection{Feature Extraction}

Feature extraction is a very important step to create the database of sign gestures. To characterize property of gesture in efficiently and effectively manner, both the global visual features and the local visual features are extracted for captured gestures. There are mainly two types of feature extraction method involved in sign recognition, first is contour based shape representation and description methods \& another is region based shape representation and description methods. Selecting of method depends on application. [8] In the proposed method, two methods are used for extracting features. Those two methods are $7 \mathrm{Hu}$ moments technique \& GLCM technique. The both methods are evaluated from every pixel of image. Using both methods, 28 parameters are evaluated from images

\subsubsection{Hu invariant moments}

$7 \mathrm{Hu}$ moments are a vector of algebraic invariants that added regular moment. They are invariant under the change of translation, size and rotation. $\mathrm{Hu}$ moments have been widely used in classification [3].7Hu moments have been developed by Mark.Hu in 1961: The formulas to calculate 7 moments are listed below.

$M_{1}=\eta_{20}+\eta_{02}$

$M_{2}=\left(\eta_{20}-\eta_{02}\right)^{2}+4 \eta_{11}^{2}$

$M_{3}=\left(\eta_{30}-3 \eta_{12}\right)^{2}+\left(3 \eta_{21}-\eta_{03}\right)^{2}$

$M_{4}=\left(\eta_{30}+\eta_{12}\right)^{2}+\left(\eta_{21}+\eta_{03}\right)^{2}$

(4)

$$
\begin{aligned}
M_{5}= & \left(\eta_{30}-3 \eta_{12}\right)\left(\eta_{30}+\eta_{12}\right)\left[\left(\eta_{30}+\eta_{12}\right)^{2}-3\left(\eta_{21}+\eta_{03}\right)^{2}\right]+ \\
& 3\left(\eta_{21}-\eta_{03}\right)\left(\eta_{21}+\eta_{03}\right)\left[3\left(\eta_{30}+\eta_{12}\right)^{2}-\left(\eta_{21}+\eta_{03}\right)^{2}\right] \\
(5) & \\
M_{6}= & \left(\eta_{20}-\eta_{02}\right)\left[\left(\eta_{30}+\eta_{12}\right)^{2}-\left(\eta_{21}+\eta_{03}\right)^{2}\right]+4 \eta_{11}\left(\eta_{30}+\right. \\
& \left.\eta_{12}\right)\left(\eta_{21}+\eta_{03}\right)
\end{aligned}
$$

$\begin{aligned} M_{7}= & \left(3 \eta_{21}-\eta_{03}\right)\left(\eta_{21}+\eta_{03}\right)\left[3\left(\eta_{30}+\eta_{12}\right)^{2}-\left(\eta_{21}+\eta_{03}\right)^{2}\right]- \\ & \left(\eta_{30}-3 \eta_{12}\right)\left(\eta_{21}+\eta_{03}\right)\left[3\left(\eta_{30}+\eta_{12}\right)^{2}-\left(\eta_{21}+\eta_{03}\right)^{2}\right]\end{aligned}$

Where,

$\eta_{\mathrm{pq}}=\mu_{\mathrm{pq}} / \mu_{00}$

All $7 \mathrm{Hu}$ moments are used as a vector to describe the image properties. The feature vectors are M1, M2, M3, M4, M5, M6, M7 stored in database.

\subsubsection{Gray Level Co-Occurrence Matrix}

The GLCM method first developed by Haralick. A gray level co-occurrence matrix is translation from two dimensional matrix of histogram. Gray level co-occurrence matrix is 
obtain by calculating how often a pixel intensity 'i' occur in spatial relationship to pixel intensity ' $j$ '. Spatial relationship is defined as a pixel to its immediate right in horizontally adjacent. In Fig 3 shown how to calculate GLCM matrix.[14]

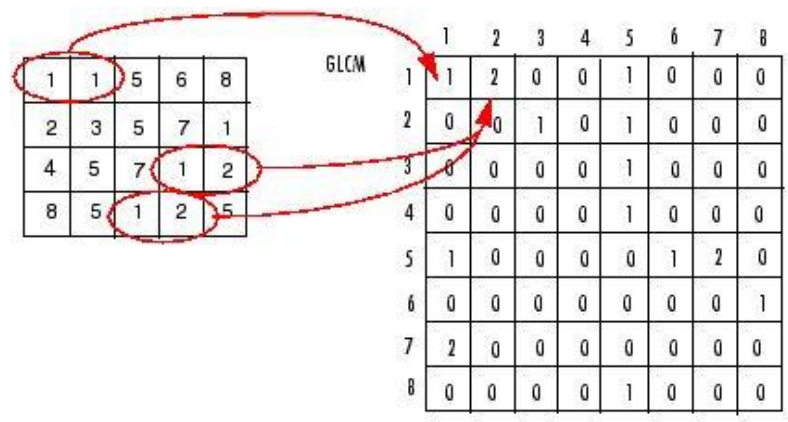

\section{Fig 3: Gray level co-occurrence matrix [14]}

Gray level co-occurrence matrix can be calculated based on different offset value. In proposed method, offset value $(2,0)$ is used for compute GLCM. Based on each computed value of GLCM, 21 features that can successfully characterize the behavior of gray level co-occurrence matrix. The following are the features calculated from co-occurrence matrix. Autocorrelation, Contrast, Correlation, Cluster Shade, Cluster Prominence, Dissimilarity, Energy, Entropy, Homogeneity, Maximum probability, Variance, Sum average, Sum variance, Sum entropy, Difference variance, Difference entropy, Information measure of correlation1, Information measure of correlation2, Inverse difference normalized, Inverse difference moment normalized, maximal correlation coefficient. Those all 21 features have their own formulas to calculate. Some of formulas are listed below.

Correlation $=\sum \sum \frac{(i-\mu x)(i-\mu y) p(i, j)}{\sigma x \sigma y}$

Entropy $=\sum \sum \frac{p(i, j)}{\log p(i, j)}$

Contrast $=\sum \sum(i-j)^{2} p(i, j)$

Variance $=\sum \sum\left(1-\sum \sum p(i, j)\right)^{2} * p(i, j)$

Inverse difference moment $=\sum \sum \frac{1}{1+(i-j)^{2}} * p(i, j)$

\subsection{Classifier}

Classification data is used as sign corresponding level with respect to groups with homogeneous characteristics, with the aim of discriminating multiple objects from each other within the image. The level is called as class. Classifier is classified in two types 1) Supervised, 2) Unsupervised. [10]. Supervised classification is dependence on the suggested method that a user can select sample values from an image that can be representative of specific classes and then applying the image processing by software to use these training samples as references for the classification of all other values in the image. Unsupervised classification is where the outcomes are dependence on the software analysis of an image without providing sample classes to user. The computer uses algorithm to check which values are related and then groups them into classes accordingly.

\subsubsection{KNN classifier}

The KNN algorithm which is known as $\mathrm{k}$ nearest neighbor algorithm. It is mostly used for character recognition. The method used for classification and regression in $\mathrm{KNN}$ classifier is non-parametric method. The output of system depends on whether KNN is used for regression or classification. While using as k-NN classification, the output is a class membership of an object. It is classified by majority values of its neighbors. The object is assigned to the class by most common among its nearest neighbors $\mathrm{k}$ ( $\mathrm{k}$ is a small positive integer) [11]. With the help of MATLAB command "knnclassify", sample vector can be classified.

$\mathrm{KNN}$ classifier is unsupervised classifier. In which some train features vector are assign to group \&after that class has been assign to input set. The following syntax used for classifier in MATLAB.

Class $=$ knnclassify (Sample, Training, Group, k, distance, rule)

Where,

Sample $=$ It is the rows which will be classified into groups. Sample set must have same number of columns as Training.

Training = Each row of training matrix must represent the row value of Group matrix. So group set must have same number of row as training.

Group = Matrix whose value represent $\&$ grouping the rows in training matrix.

$\mathrm{K}=$ The default value is 1 . It is nearest neighbors used in the classification. This value should be greater than 1 .

Distance $=$ It will be any one of the following methods. But default it takes 'Euclidean' distance. Methods are Euclidean, cityblock, cosine, correlation, hamming

Rule $=$ This is used to decide that how to classify the Sample Matrix. 'Nearest rule' is default rule used in 'knnclassify'. Other rules are nearest, random, consensus

\subsubsection{PNN classifier}

The abbreviation of PNN is Probabilistic Neural Network. It is neural network based classifier. It is mostly used in Pattern recognition \& classification. It is based on feed-forward neural network. This is supervised classifier which is used to train the network and next step is to testing the sample vector. The MATLAB command 'newpnn' is used to create the network \& train the network'. In this command, input vector $\&$ class vector is needed to train the network. After this, Sample vector is simulated using simulate command.

The following MATLAB command used for PNN classifier

$$
\begin{aligned}
& \text { 1. net }=\text { newpnn(P,T) } \\
& \text { 2. } \mathrm{y}=\operatorname{sim}(\text { net }, \mathrm{S})
\end{aligned}
$$

\subsection{Text \& Speech}

Now final step is to display a recognize character or word \&speech output is given to speaker from audio database in both Hindi and English languages. English text is easily displayed using MATLAB inbuilt function. For English sound, the function called as system.Speech.Synthesis.SpeechSynthesizer is used. Now major task is to implement Hindi text \&Hindi speech. MATLAB doesn't support Hindi character so Hindi text is displayed by creating database of each alphabet with the help 
of images of individual alphabet \& display respectively. Similarly, the database of Hindi speech is made of audio files for each alphabet. To generate Hindi sound file .wav format is used as it is give very good sound quality.

\section{RESULT}

The proposed system is implementing on MATLAB software. As mentioned previously that in this paper not only characters but also words are recognized. For both English and Hindi languages are considered. Indian sign language consists of some words with only single gesture which are common and use in day to day life. For example stop, hello, weekdays and etc. So considering above parameter result is mainly categorized into 3 categories. Those categories are listed below.
A. English alphabets
B. Hindi alphabets
C. Single word with single gestures (SWSG)

The following are the step wise output of each category. Same procedure is followed for each category with minor changes. So each category consists of captured image, binary images, features values, comparison of classifier parameter and finally recognized output. Speech has an audio output so it is unable to cover in this paper as a result.

\subsection{English Alphabets}

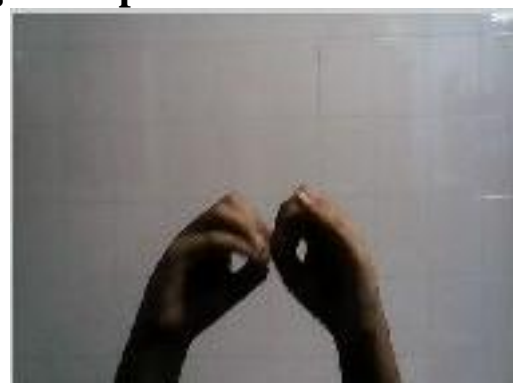

Fig 4: Captured Gesture for Alphabet 'B'

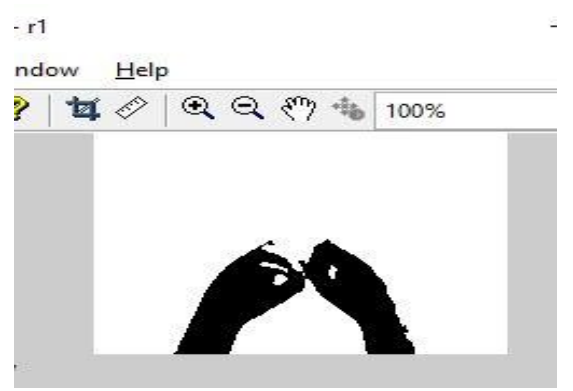

Fig 5: Binary conversion of captured gesture

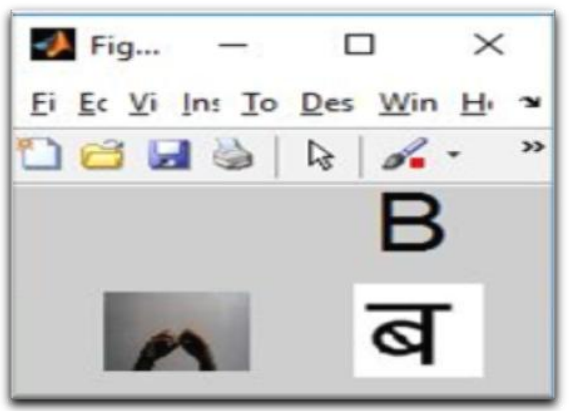

Fig 6: Recognize output for English character 'B'
Fig 4 shows the captured gesture for English alphabet ' $B$ ' from web camera of computer. Now with help MATLAB function captured gesture is converted into binary image as shown in Fig 5.

Next step is to evaluate a feature values using feature extraction methods. Table 1. Shows 7 features values using $7 \mathrm{Hu}$ moment techniques \& Table 2. Shows 21 feature values calculated from gray level co-occurrence matrix. So total 28 feature values are evaluated of each image. Using data base for all letters, we can easily recognize the capture gesture with the help of classifier. Fig 6 Shows recognize output for captured gesture. In output captured gesture, recognize letter in Hindi and English languages are shown.

\subsection{Hindi Alphabets}

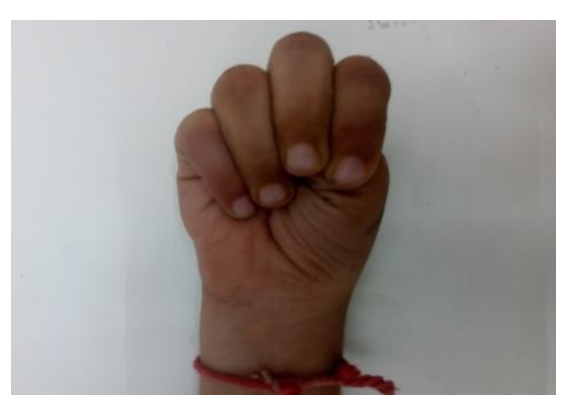

Fig 7: Captured Gesture for Hindi alphabet ' $ত$ '

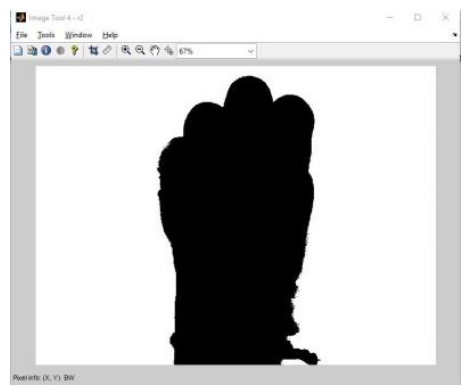

Fig 8: Binary conversion of captured gesture 'उ'

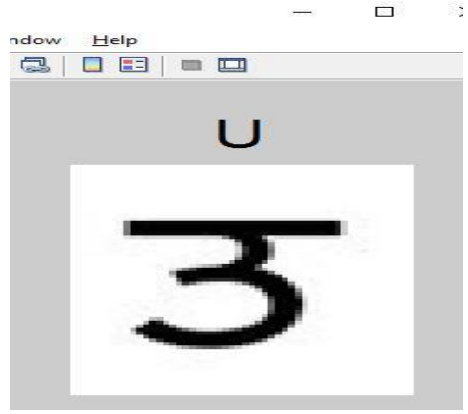

Fig 9: Recognize output for Hindi character ' $\checkmark$ '

\subsection{Single word with single gestures (SWSG)}

In single word with single gesture, seven days of week has been considered and as result one day is shown. 


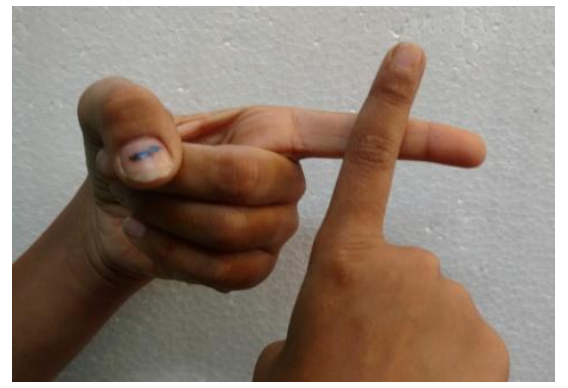

Fig 10: Captured gesture for word 'Saturday'

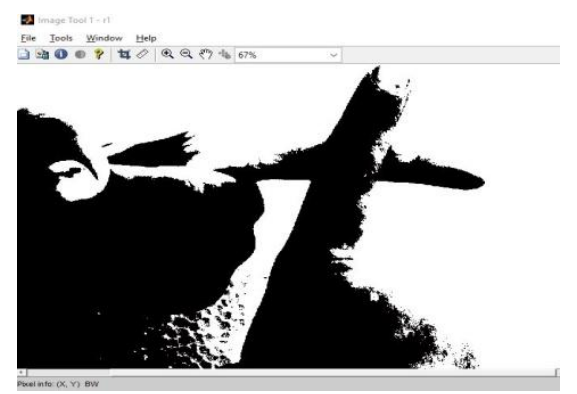

Fig 11: Binary Image of captured gesture 'Saturday'

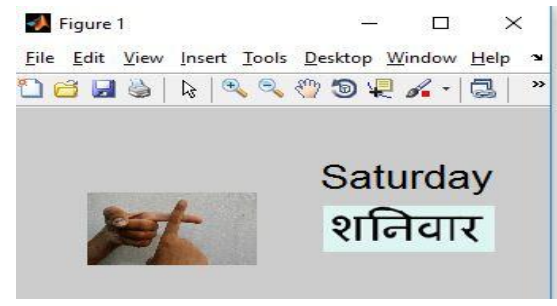

Fig 12: Recognize output for word 'Saturday'

TABLE 1.Calculatedfeatures value using 7Hu moment technique for Character / Words

\begin{tabular}{|c|c|c|c|}
\hline \multirow{2}{*}{ Moment } & \multicolumn{3}{|c|}{ Character / Words } \\
\cline { 2 - 4 } & $\mathrm{B}$ & उ & Monday \\
\hline M1 & 0.203813 & 0.492023 & 0.3417 \\
\hline M2 & 0.004242 & 0.052286 & 0.087485 \\
\hline M3 & $3.13 \mathrm{E}-05$ & 0.001627 & 0.01047 \\
\hline M4 & $6.16 \mathrm{E}-05$ & 0.002 & 0.002108 \\
\hline M5 & $-1.5 \mathrm{E}-10$ & $5.3 \mathrm{E}-07$ & $-3.7 \mathrm{E}-06$ \\
\hline M6 & $-3.7 \mathrm{E}-07$ & $3.51 \mathrm{E}-05$ & 0.000297 \\
\hline M7 & $8.38 \mathrm{E}-10$ & $-1.1 \mathrm{E}-06$ & $9.26 \mathrm{E}-06$ \\
\hline
\end{tabular}

TABLE 2.Calculated features value from gray level cooccurrence matrix (GLCM) for Character / Words

\begin{tabular}{|c|c|c|c|}
\hline \multirow{2}{*}{ Moment } & \multicolumn{3}{|c|}{ Character / Words } \\
\hline & B & उ & Monday \\
\hline Autocorrelation & 17.18559 & 27.53655 & 23.82297 \\
\hline Contrast & 0.070195 & 0.143958 & 0.071916 \\
\hline $\begin{array}{l}\text { Correlation: } \\
\text { MATLAB }\end{array}$ & 0.976243 & 0.986028 & 0.979531 \\
\hline Correlation & 0.976243 & 0.986028 & 0.979531 \\
\hline $\begin{array}{c}\text { Cluster } \\
\text { Prominence }\end{array}$ & 134.2255 & 675.7224 & 100.6514 \\
\hline Cluster Shade & -12.9696 & 3.823047 & -9.43755 \\
\hline Dissimilarity & 0.057309 & 0.140161 & 0.070654 \\
\hline Energy & 0.269031 & 0.106684 & 0.253808 \\
\hline Entropy & 1.667406 & 2.493145 & 1.726773 \\
\hline $\begin{array}{l}\text { Homogeneity: } \\
\text { MATLAB }\end{array}$ & 0.973211 & 0.930538 & 0.964876 \\
\hline Homogeneity & 0.972632 & 0.930299 & 0.964799 \\
\hline $\begin{array}{l}\text { Maximum } \\
\text { probability }\end{array}$ & 0.388556 & 0.163461 & 0.412676 \\
\hline Sum of squares & 17.12461 & 27.46979 & 23.71777 \\
\hline Sum average & 7.935892 & 9.477798 & 9.402599 \\
\hline Sum variance & 45.80119 & 70.76337 & 66.68406 \\
\hline Sum entropy & 1.613947 & 2.385416 & 1.674136 \\
\hline $\begin{array}{l}\text { Difference } \\
\text { variance }\end{array}$ & 0.070195 & 0.143958 & 0.071916 \\
\hline Difference entropy & 0.221448 & 0.411684 & 0.257207 \\
\hline $\begin{array}{c}\text { Information } \\
\text { measure of } \\
\text { correlation1 }\end{array}$ & -0.83463 & -0.76313 & -0.80998 \\
\hline $\begin{array}{c}\text { Information } \\
\text { measure of } \\
\text { correlation2 }\end{array}$ & 0.953021 & 0.976673 & 0.951173 \\
\hline $\begin{array}{c}\text { Inverse difference } \\
\text { moment } \\
\text { normalized }\end{array}$ & 0.993769 & 0.984468 & 0.992163 \\
\hline
\end{tabular}

The performance of proposed classifier is decided based on some performance evaluation parameter which is calculated based on Actual Classification \& Predicted Classification. The formulas are used to calculate parameter such as classified 
rate, error rate, sensitivity, positive likelihood, positive predicated value etc.

TABLE 3. Comparison between KNN \& PNN classifier based on Performance evaluation Parameter

\begin{tabular}{|c|c|c|}
\hline Parameter & KNN & PNN \\
\hline Classified Rate & 0.85 & 0.89 \\
\hline Error Rate & 0.15 & 0.11 \\
\hline Prevalance & 0.432 & 0.458 \\
\hline Sensitivity & 0.8388 & 0.8699 \\
\hline Specificity & 0.8966 & 0.922 \\
\hline Positive predictive value & 0.84 & 0.93 \\
\hline Negative predictive value & 0.8248 & 0.87 \\
\hline Positive Likelihood & 8.2469 & 9.84 \\
\hline Negative Likelihood & 0.142 & 0.139 \\
\hline
\end{tabular}

\section{CONCLUSION \& FUTURE WORK}

Using two methods, 7Hu moment techniques \& gray level cooccurrence matrix for feature extraction strong database has been implemented. KNN \& PNN classifier are compared using many performance parameter \& conclude that PNN classifier is better. Considering all categories average more than $84 \%$ accuracy is achieved. English Speech has very good Quality because of inbuilt MATLAB function while Hindi speech is pre-recorded sound file saved in database therefore quality depends on recorded sound file. In this method .wav audio file format is used for Hindi speech. In future work ISL can be converted into other Indian languages such as Marathi, Gujarati, and various Indian languages. We can also convert and translate other Indian sign language. Considering time parameter, this concept for video conferencing with deaf \& dumb people for advanced communication. Even it can apply on the smartphone platform, due to its easy use, mobility and tremendous growth.

\section{ACKNOWLEDGMENTS}

It is nice pleasure of large satisfaction on behalf of me to specific my feeling to my guide Prof. Aarti G. Ambekar, whose constant encouragement enabled to figure out the problems. Her continuous motivation, patience and experience in discussion throughout progress of my work have benefited to extent.

\section{REFERENCES}

[1] Cheok Ming Jin, Zaid Omar \& Mohamed Hisham Jaward, "A Mobile Application of American Sign Language Translation via Image Processing Algorithms" 2016 IEEE Region 10 Symposium (TENSYMP), Bali,
Indonesia

[2] Rohit Rastogi ,Shashank Mittal \& Sajan Agarwal “ Novel Approach for Communication among Blind, Deaf and Dumb People" 2015 2nd International Conference on Computing for Sustainable Global Development

[3] Suganya R \& Dr. T.Meeradevi "Design Of a Communication aid for physically challenged" Ieee Sponsored 2nd International Conference On Electronics And Communication System (ICECS 2015)

[4] Kusurnika Krori Dutta, Satheesh Kumar Raju, Anil Kumar G, Sunny Arokia Swarny "Double Handed Indian Sign Language to Speech and Text" 2015 Third International Conference on Image Information Processing

[5] Piotr Muzyka, Marek Frydrysick \& Elzbieta Roszkowska "Real-Time Detection of Hand Gestures".

[6] Priyanka C Pankajakshan \& Thilagavathi B "Sign Language Recognition system" IEEE Sponsored 2nd International conference ICIIECS'15

[7] Prof. Prashant G. Ahire, Kshitija B. Tilekar, Tejaswini A. Jawake, Pramod B. Warale "Two Way Communicator Between Deaf and Dumb People And Normal People". 2015 International Conference on Computing Communication Control and Automation

[8] Ashish s. Nikam \& Aarti G. Ambekar "Bilingual Sign Recognition Using Image Based Hand Gesture Technique for Hearing and Speech Impaired People" IEEE $2^{\text {nd }}$ International conference on Computing, Communication, Control \& Automation. Aug 2016.

[9] Ashish s. Nikam \& Aarti G. Ambekar "Sign language recongization using image based hand gesture recongnition techniques" IEEE $3^{\text {rd }}$ International conference on Innovation in Information, Embedded \& Communication system (ICIIECS'16).

[10] P. Suresh, N. Vasudevan, and N. Ananthanarayanan, "Computer-aided interpreter for hearing and speech impaired," Proc. IEEE 4th Int. Con! Comput. IntelI. Commun. Syst. Networks ,CICSyN2012, pp. 248-253.

[11] A. S. Ghotkar, R. Khatal, S. Khupase, S. Asati, and M. Hadap, "Hand gesture recognition for Indian Sign Language," IEEE Int. Conf Comput. Commun. Informatics 2012, pp. 1-4.

[12] A. Thorat, V. Satpute, A. Nehe, T.Atre Y.Ngargoje , "Indian Sign Language Recognition System for Deaf People " Int. J.Adv. compt.comm. engg.IJARCCE,pp.5319-5321,2014

[13] Yang quan, Peng jinye, Li yulong"Chinese Sign Language Recognition Based on Gray-Level CoOccurrence Matrix and Other Multi-features Fusion"ICIEA 2009, pp 1569-1572.

[14] MATLAB/help 